Pure and Applied Mathematics Quarterly

Volume 3, Number 2

(Special Issue: In honor of

Leon Simon, Part 1 of 2)

$595-613,2007$

\title{
Heat Flow of Biharmonic Maps in Dimensions Four and Its Application
}

\author{
Changyou Wang
}

\begin{abstract}
Let $(M, g)$ be a four dimensional compact Riemannian manifold without boundary, $(N, h) \subset \mathbf{R}^{k}$ be a compact Riemannian submanifold without boundary. We establish the existence of a global weak solution to the heat flow of extrinsic biharmonic maps from $M$ to $N$, which is smooth away from finitely many singular times. As a consequence, we prove that if $\Pi_{4}(N)=\{0\}$, then any free homotopy class $\alpha \in[M, N]$ contains at least one minimizing biharmonic map.
\end{abstract}

$\S 1$. Introduction

Let $(M, g),(N, h)$ be smooth compact Riemannian manifolds without boundaries. Assume that $(N, h)$ is isometrically embedded into an Euclidean space $\mathbf{R}^{k}$. For a non-negative integer $l$, and $1 \leq p<+\infty$, the Sobolev space $W^{l, p}(M, N)$ is defined by

$$
W^{l, p}(M, N)=\left\{u \in W^{l, p}\left(M, \mathbf{R}^{k}\right) \mid u(x) \in N \text { for a.e. } x \in M\right\} .
$$

On $W^{2,2}(M, N)$, there are two natural, second order energy functionals:

$$
H(u)=\int_{M}|\Delta u|^{2} d v_{g}, \quad T(u)=\int_{M}|\tau(u)|^{2} d v_{g},
$$

where $\Delta$ is the Laplace-Beltrami operator of $(M, g), d v_{g}$ is the volume element of $(M, g), \tau(u)=(\Delta u)^{T}:=\Delta u+A(u)(\nabla u, \nabla u)$ is the tension field of $u$, and $A(\cdot)(\cdot, \cdot)$ is the second fundamental form of $(N, h)$ in $\mathbf{R}^{k}$.

Recall that a map $u \in W^{2,2}(M, N)$ is called an extrinsic (or intrinsic, resp.) biharmonic map if $u$ is a critical point of $H(\cdot)$ (or $T(\cdot)$, resp.). For a sufficiently small $\delta>0$, let $\Pi: N_{\delta} \rightarrow N$ be the smooth nearest point projection map,

Received February 3, 2006. 
$P(y)=\nabla \Pi(y): \mathbf{R}^{k} \rightarrow T_{y} N$ be the orthogonal projection to the tangent space at $y \in N$. Note that

$$
A(y)(X, Y)=\nabla_{X} P(y)(Y), \forall y \in N, X, Y \in T_{y} N .
$$

It is readily seen (cf. Wang $[\mathrm{W} 1,2])$ that the Euler-Lagrange equation for extrinsic biharmonic maps is

$$
\Delta^{2} u=\Delta(A(u)(\nabla u, \nabla u))+\langle\Delta u, \Delta(P(u))\rangle+2\langle\nabla \Delta u, \nabla(P(u))\rangle .
$$

(1.1) is equivalent to the geometric form:

$$
\Delta^{2} u \perp T_{u} N,
$$

in the sense of distributions.

Regularity issues for biharmonic maps have first been studied by Chang-WangYang [CWY] for spheres $N=\mathbf{S}^{k-1}$, and later by Wang [W1,2] for general manifolds $N$ (see also [W3] and Strzelecki [P]).

Motivated by the heat flow of harmonic maps from surfaces (see Struwe [S] and Sacks-Uhlenbeck [SaU]), and the problem by Eells-Lemaire [EL] that is to find extrinsic (or intrinsic) biharmonic maps among any free homotopy class $\alpha \in[M, N]$ for $\operatorname{dim}(M)=4$, people are interested in the study of the heat flow of biharmonic maps $u: M \times \mathbf{R}_{+} \rightarrow N$ :

$$
\begin{aligned}
u_{t}+\Delta^{2} u & =\Delta(A(u)(\nabla u, \nabla u))+\langle\Delta u, \Delta(P(u))\rangle) \\
& +2\langle\nabla \Delta u, \nabla(P(u))\rangle, M \times \mathbf{R}_{+}, \\
u(x, 0) & =\phi(x), x \in M,
\end{aligned}
$$

where $\phi \in W^{2,2}(M, N)$ is a given map.

For a smooth map $\phi \in C^{\infty}(M, N)$, the short time existence of smooth solutions to (1.3)-(1.4) is well-known, since (1.3) is a fourth order strongly parabolic system (see Lamm [L1] for more details). Moreover, if $\operatorname{dim} M \leq 3$, then such a short time smooth solution can be extended to be a globally smooth solution. For dim $M \geq 4$, the short time smooth solution may develop a singularity at finite time. For $\operatorname{dim} M=4$, Lamm [L2] proved that (1.3)-(1.4) has a globally smooth solution $u \in C^{\infty}\left(M \times \mathbf{R}_{+}, N\right)$, provided that $\phi \in C^{\infty}(M, N)$ has small Hessian energy $H(\phi)$. Without the smallness assumption, we establish a partially smooth, weak solution to (1.3)-(1.4). More precisely, we have

Theorem A. For $\operatorname{dim} M=4$ and any map $\phi \in W^{2,2}(M, N)$, there exists a global weak solution $u: M \times \mathbf{R}_{+} \rightarrow N$ of (1.3)-(1.4) satisfying:

(1) For any $0<T<+\infty$,

$$
\int_{0}^{T} \int_{M}\left|u_{t}\right|^{2} d v_{g} d t+\int_{M}|\Delta u|^{2}(\cdot, T) d v_{g} \leq \int_{M}|\Delta \phi|^{2} d v_{g}
$$

and $H(u(\cdot, t))$ is monotonically nonincreasing with respect to $t \geq 0$. 
(2) There exist an $\epsilon_{0}>0$, a positive integer $L$ depending only on $\phi, M, N$, and $0<t_{1}<\cdots<t_{L}<+\infty$ such that $u \in C^{\infty}\left(M \times\left(\mathbf{R}_{+} \backslash \cup\left\{t_{1}, \cdots, t_{L}\right\}\right), N\right)$, and

$$
\mathcal{E}\left(u, t_{i}\right):=\lim _{r \downarrow 0} \limsup _{t \uparrow t_{i}} \sup _{x \in M} \int_{B_{r}(x)}|\Delta u|^{2} d v_{g} \geq \epsilon_{0}^{2}, 1 \leq i \leq L .
$$

(3) The quantity $\epsilon_{0}$ can be characterized by

$\epsilon_{0}^{2}=\inf \left\{\int_{\mathbf{R}^{4}}|\Delta \omega|^{2} d x \mid \omega \in C^{\infty} \cap W^{2,2}\left(\mathbf{R}^{4}, N\right)\right.$ nonconstant biharmonic maps $\}$.

(4) For $1 \leq i \leq L$, there exist a nonconstant biharmonic map $\omega_{i} \in C^{\infty} \cap$ $W^{2,2}\left(\mathbf{R}^{4}, N\right), t_{j_{i}} \uparrow t_{i},\left\{x_{j_{i}}\right\} \subset M$ with $x_{j_{i}} \rightarrow x_{i}, r_{j_{i}} \rightarrow 0$, such that

$$
u_{j_{i}}(\cdot)=u\left(x_{j_{i}}+r_{j_{i}}, t_{j_{i}}\right) \rightarrow \omega_{i}, \quad \text { in } C_{l o c}^{k}\left(\mathbf{R}^{4}\right) \forall k \geq 1 .
$$

Remark 1.1. After the submission of the paper, we have learned that Theorem A has also been independently proved by Gastel [G] recently.

Remark 1.2. It is an open question whether the weak solution as in theorem $A$ has at most finitely many singularities (i.e. at most finitely many singular points in each singular time).

Remark 1.3. Motivated by the existence theory of the heat flow of harmonic maps in high dimensions by Chen-Struwe [CS], it is a very interesting problem to study the heat flow of biharmonic maps in dimensions at least five. The main difficulty is the lack of suitable monotonicity formulars of parabolic types. However, in a forthcoming article [W4], we are able to establish the existence of smooth solutions to (1.3)-(1.4), under the assumption that $\operatorname{dim} M \leq 8$ and $H(\phi)$ is sufficiently small.

Remark 1.4. It is also a very natural question to study the heat flow of intrinsic biharmonic maps. In [L3], Lamm proved the existence of smooth solutions to the heat flow of intrinsic biharmonic maps provided that $\operatorname{dim} M=4$ and $N$ has non-positive sectional curvature. However, without this condition on curvature it seems difficult to obtain a global, weak solution due to the lack of coercivity property of $T(\cdot)$.

Remark 1.5. The fourth order heat flow has been recently employed by KuwertSchätzle $[\mathrm{KS} 1,2]$ in the study of Willmore functionals. Some earlier existence results on Willmore surfaces were established by Simon [Sl]. The interested readers may find that there are similar analytic techniques between the Willmore flow and the heat flow of biharmonic maps studied here. In particular, the integral estimate and interpolation inequalities are common themes. 
As a consequence of theorem A, we prove

Theorem B. If $\operatorname{dim} M=4$ and $\Pi_{4}(N)=\{0\}$, then any free homotopy class $\alpha \in[M, N]$ contains at least one biharmonic map $u \in C^{\infty}(M, N)$ that minimizes the Hessian energy, i.e. $H(u)=\min \left\{H(v) \mid v \in C^{\infty}(M, N),[v]=\alpha\right\}$.

The paper is written as follows. In $\S 2$, we recall density of $C^{\infty}(M, N)$ in $W^{2,2}(M, N)$ (see, Brezis-Nirenberg [BN]) and prove the quantization effect (1.7). In $\S 3$, we review some integral estimates for smooth solutions of (1.3) by Lamm [L2], the characterization of the first singular time, and prove Theorem A. In $\S 4$, we give a proof of Theorem B.

Acknowledgement. The author thanks the referee for his careful readings and many helpful suggestions. This work is partially supported by NSF grant 0400718 .

$\S 2$. Density of smooth maps and quantization effect (1.7)

In this section, we establish a Bochner type inequality and an $\epsilon$-gradient estimate for smooth biharmonic maps and prove the quantization fact (1.7). We also recall a well-known fact (see, [BN]) on the density of smooth maps in $W^{2,2}(M, N)$ whenever $\operatorname{dim} M=4$. First we have

Proposition 2.1. There are $C_{1}, C_{2}>0$ depending only on $N$ such that if $u \in C^{\infty}\left(\mathbf{R}^{n}, N\right)$ is an (extrinsic) biharmonic map, then $e(u) \equiv|\Delta u|^{2}+C_{1}|\nabla u|^{4}$ satisfies

$$
\Delta e(u)+C_{2} e(u)(1+e(u)) \geq 0, \text { in } \mathbf{R}^{n} .
$$

Proof. Note $(\Delta u)^{T}=P(u)(\Delta u)$ and $\Delta u=(\Delta u)^{T}+A(u)(\nabla u, \nabla u)$. It follows from (1.2) that we have $\left\langle\Delta^{2} u,(\Delta u)^{T}\right\rangle=0$. Hence

$$
\Delta\left(|\Delta u|^{2}\right)=2\left\langle\Delta^{2} u, \Delta u\right\rangle+2|\nabla \Delta u|^{2}=2\left\langle\Delta^{2} u, A(u)(\nabla u, \nabla u)\right\rangle+2|\nabla \Delta u|^{2} .
$$

This and (1.1) imply

$$
\begin{aligned}
\Delta\left(|\Delta u|^{2}\right) & =2|\nabla \Delta u|^{2}+2\langle\Delta(A(u)(\nabla u, \nabla u)), A(u)(\nabla u, \nabla u)\rangle \\
& +2\langle\langle\nabla \Delta u, \nabla(P(u))\rangle, A(u)(\nabla u, \nabla u)\rangle \\
& +\langle\langle\Delta(P(u)), \Delta u\rangle, A(u)(\nabla u, \nabla u)\rangle \\
& =2|\nabla \Delta u|^{2}+I I+I I I+I V .
\end{aligned}
$$

It is easy to see that there exist $C_{3}, C_{4}$ depending only on $N$ such that

$$
|I I I| \leq 2\|\nabla P\|_{L^{\infty}(N)}\|A\|_{L^{\infty}(N)}|\nabla \Delta u \| \nabla u|^{3} \leq \frac{1}{2}|\nabla \Delta u|^{2}+C_{3}\left(|\nabla u|^{4}+|\nabla u|^{8}\right),
$$


and

$$
\begin{aligned}
|I V| & \leq\|A\|_{L^{\infty}(N)}|\Delta(P(u)) \| \Delta u||\nabla u|^{2} \\
& \leq\|A\|_{L^{\infty}(N)}|\Delta u||\nabla u|^{2}\left(\|\nabla P\|_{L^{\infty}(N)}|\Delta u|+\left\|\nabla^{2} P\right\|_{L^{\infty}(N)}|\nabla u|^{2}\right) \\
& \leq C_{4}\left(|\Delta u|^{2}|\nabla u|^{2}+|\Delta u \| \nabla u|^{4}\right) \\
& \leq C_{4}\left(|\Delta u|^{2}+|\Delta u|^{4}+|\nabla u|^{4}+|\nabla u|^{8}\right) .
\end{aligned}
$$

To estimate $I I$, we first calculate $\Delta(A(u)(\nabla u, \nabla u))$ as follows.

$$
\begin{aligned}
\Delta(A(u)(\nabla u, \nabla u)) & =\nabla^{2} A(u)(\nabla u, \nabla u)(\nabla u, \nabla u)+\nabla A(u)(\nabla u, \nabla u)(\Delta u) \\
& +4 \nabla A(u)\left(\nabla^{2} u, \nabla u\right)(\nabla u)+2 A(u)\left(\nabla^{2} u, \nabla^{2} u\right) \\
& +2 A(u)(\nabla \Delta u, \nabla u) .
\end{aligned}
$$

This implies that there exists a $C_{5}$ depending only on $N$ such that

$$
|\Delta(A(u)(\nabla u, \nabla u))| \leq C_{5}\left(|\nabla u|^{4}+\left|\nabla^{2} u\right|^{2}+|\nabla \Delta u||\nabla u|+\left|\nabla^{2} u\right||\nabla u|^{2}\right) .
$$

Therefore we have

$$
\begin{aligned}
|I I| & \leq|A(u)(\nabla u, \nabla u)||\Delta(A(u)(\nabla u, \nabla u))| \\
& \leq C_{5}\|A\|_{L^{\infty}(N)}\left(|\nabla \Delta u||\nabla u|^{3}+\left|\nabla^{2} u\right||\nabla u|^{4}\right. \\
& \left.+|\nabla u|^{6}+\left|\nabla^{2} u\right|^{2}|\nabla u|^{2}\right) .
\end{aligned}
$$

By the Hölder inequality, we have, for some $C_{6}$ depending only on $N$,

$$
\begin{gathered}
|\nabla u|^{6} \leq|\nabla u|^{4}+|\nabla u|^{8}, \\
\|A\|_{L^{\infty}(N)}|\nabla \Delta u||\nabla u|^{3} \leq \frac{1}{2 C_{5}}|\nabla \Delta u|^{2}+C_{6}\left(|\nabla u|^{4}+|\nabla u|^{8}\right),
\end{gathered}
$$

and

$$
\left|\nabla^{2} u\right||\nabla u|^{4} \leq\left|\nabla^{2} u\right|^{2}|\nabla u|^{2}+|\nabla u|^{6} \leq\left|\nabla^{2} u\right|^{2}|\nabla u|^{2}+|\nabla u|^{4}+|\nabla u|^{8} .
$$

Putting these inequalities together, we obtain

$$
|I I| \leq \frac{1}{2}|\nabla \Delta u|^{2}+C_{6}\left[\left(|\nabla u|^{4}+|\nabla u|^{8}\right)+\left|\nabla^{2} u\right|^{2}|\nabla u|^{2}\right]
$$

Putting (2.4)-(2.7) into (2.3), we have, for some $C_{7}$ depending only on $N$,

$$
\Delta\left(|\Delta u|^{2}\right) \geq|\nabla \Delta u|^{2}-C_{7}\left(|\Delta u|^{2}+|\nabla u|^{4}\right)\left(1+\left(|\Delta u|^{2}+|\nabla u|^{4}\right)\right)-C_{7}\left|\nabla^{2} u\right|^{2}|\nabla u|^{2} \text {. }
$$

On the other hand, direct calculations imply

$$
\begin{aligned}
\Delta\left(|\nabla u|^{4}\right) & =8\left|\left\langle\nabla u, \nabla^{2} u\right\rangle\right|^{2}+4|\nabla u|^{2}\left|\nabla^{2} u\right|^{2}+4|\nabla u|^{2}\langle\nabla u, \nabla \Delta u\rangle \\
& \geq 4\left|\nabla^{2} u\right|^{2}|\nabla u|^{2}-C_{7}^{-1}|\nabla \Delta u|^{2}-C_{7}\left(|\nabla u|^{4}+|\nabla u|^{8}\right) .
\end{aligned}
$$

Therefore, if we choose $C_{1}=C_{7}$, then there exists $C_{2}>0$ depending only on $N$ such that

$$
\Delta\left(|\Delta u|^{2}+C_{1}|\nabla u|^{4}\right) \geq-C_{2}\left(|\Delta u|^{2}+C_{1}|\nabla u|^{4}\right)\left[1+\left(|\Delta u|^{2}+C_{1}|\nabla u|^{4}\right)\right] .
$$

This yields (2.1). 
Now we prove an $\epsilon_{0}$-gradient estimate for biharmonic maps.

Theorem 2.2. For $n \geq 4$, there exists an $\epsilon_{0}>0$ depending only on $N$ such that if $u \in C^{\infty}\left(\mathbf{R}^{n}, N\right)$ is an extrinsic biharmonic map and satisfies, for some $B_{R} \subset \mathbf{R}^{n}$,

$$
R^{4-n} \int_{B_{R}}\left(\left|\nabla^{2} u\right|^{2}+|\nabla u|^{4}\right) \leq \epsilon_{0}^{2}
$$

then, for any $k \geq 1$,

$$
r^{k} \max _{y \in B_{r}(x)}\left|\nabla^{k} u\right|(y) \leq C\left(k, \epsilon_{0}\right), \quad \forall x \in B_{\frac{R}{4}}, \quad r \leq \frac{R}{4} .
$$

Proof. We remark that the argument to prove partial regularity for stationary biharmonic maps by [CWY] and [W2] also yields a proof of theorem 2.2. Here we give a direct proof that is based on Proposition 2.1, that is similar to that by Schoen $[\mathrm{Sr}]$ on smooth harmonic maps.

For simplicity, we assume $x=0$. Since $u$ is a $C^{\infty}$-biharmonic map, it is a stationary biharmonic map (see [CWY] and [W1,2] for the definition). Hence it follows [W2] Lemma 5.2 and Lemma 5.3 that there exists a $\theta_{0} \in(0,1)$ such that

$$
s^{4-n} \int_{B_{s}(x)}\left(\left|\nabla^{2} u\right|^{2}+|\nabla u|^{4}\right) \leq C \epsilon_{0}^{2}, \forall B_{s}(x) \subset B_{2 \theta_{0} R} .
$$

Denote $R_{0}=\theta_{0} R$ and $e(u)=|\Delta u|^{2}+C_{1}|\nabla u|^{4}$ with $C_{1}$ the same constant as in Proposition 2.1. Then there exists $r_{0} \in\left[0, R_{0}\right)$ such that

$$
\left(R_{0}-r_{0}\right)^{4} \max _{B_{r_{0}}} e(u)=\max _{0 \leq s \leq R_{0}}\left(R_{0}-s\right)^{4} \max _{B_{s}} e(u) .
$$

Moreover, there exists a $x_{0} \in B_{r_{0}}$ such that

$$
e_{0}=e(u)\left(x_{0}\right)=\max _{B_{r_{0}}} e(u) .
$$

Set $\rho_{0}=\frac{1}{2}\left(R_{0}-r_{0}\right)$. Then we have

$$
\max _{B_{\rho_{0}}\left(x_{0}\right)} e(u) \leq \max _{B_{r_{0}+\rho_{0}}} e(u) \leq \frac{\left(R_{0}-r_{0}\right)^{4} e_{0}}{\left(R_{0}-\left(r_{0}+\rho_{0}\right)\right)^{4}}=16 e_{0} .
$$

Set $\delta_{0}=e_{0}^{\frac{1}{4}} \rho_{0}$. We need to show $\delta_{0} \leq 1$. For otherwise, we have $\delta_{0}>0$ or $e_{0}^{-\frac{1}{4}}<\rho_{0}$. Let $v \in C^{\infty}\left(B_{\delta_{0}}, N\right)$ be defined by

$$
v(y)=u\left(x_{0}+\frac{y}{e_{0}^{\frac{1}{4}}}\right), y \in B_{\delta_{0}} .
$$

Then $v$ is an extrinsic biharmonic map, and (2.15) implies

$$
\max _{B_{\delta_{0}}} e(v) \leq 16, \quad e(v)(0)=1 .
$$


Therefore Proposition 2.1 implies

$$
\Delta e(v)+C e(v) \geq 0, \quad \text { in } B_{\delta_{0}} .
$$

Hence, by the Harnack inequality (see [GT] Theorem 8.17, page 184), we conclude

$$
1=e(v)(0) \leq C \int_{B_{1}} e(v)(y) d y .
$$

On the other hand, by rescalings, we have

$$
\int_{B_{1}} e(v)(y) d y=\left(e_{0}^{-\frac{1}{4}}\right)^{4-n} \int_{B_{e_{0}^{-\frac{1}{4}}}\left(x_{0}\right)} e(u)(x) d x \leq C \epsilon_{0}^{2},
$$

where it used that

$e_{0}^{-\frac{1}{4}} \leq \rho_{0}=\frac{1}{2}\left(R_{0}-r_{0}\right) \leq R_{0}$ and (2.12). (2.19) contradicts with (2.18), provided that $\epsilon_{0}$ is chosen to be sufficiently small. Hence $\delta_{0}=e_{0}^{\frac{1}{4}} \rho_{0} \leq 1$ and (2.13) implies

$$
\left(\frac{\theta_{0} R}{2}\right)^{4} \max _{\frac{B_{0} R}{2}} e(u) \leq 16 .
$$

This, combined with simple covering arguments, yields (2.11) for $k=2$. (2.11) for $k \geq 3$ can be deduced by the standard theory of 4 th order linear elliptic equations.

As a direct consequence, we can prove the following quantization result, which was previously proved in [L2] (Theorem 1.1) by a different method.

Corollary 2.3. There exists $\epsilon_{0}>0$ depending only on $N$ such that $\epsilon_{0}^{2}:=\inf \left\{\int_{\mathbf{R}^{4}}|\Delta \omega|^{2}, \omega \in C^{\infty} \cap W^{2,2}\left(\mathbf{R}^{4}, N\right)\right.$ nonconstant biharmonic maps $\}>0$.

Proof. Suppose (2.20) were false. Then there exist a sequence of nonconstant extrinsic biharmonic maps $\left\{\omega_{k}\right\} \subset C^{\infty} \cap W^{2,2}\left(\mathbf{R}^{4}, N\right)$ such that

$$
\int_{\mathbf{R}^{4}}\left|\Delta \omega_{k}\right|^{2} \leq k^{-1}
$$

Since $\omega_{k} \in W^{2,2}\left(\mathbf{R}^{4}, N\right)$, we have, by integration of parts,

$$
\int_{\mathbf{R}^{4}}\left|\nabla^{2} \omega_{k}\right|^{2}=\int_{\mathbf{R}^{4}}\left|\Delta \omega_{k}\right|^{2} \leq k^{-1} .
$$

This, combined with the Sobolev embedding inequality, implies

$$
\int_{\mathbf{R}^{4}}\left|\nabla \omega_{k}\right|^{4} \leq C\left(\int_{\mathbf{R}^{4}}\left|\nabla^{2} \omega_{k}\right|^{2}\right)^{2} \leq C k^{-2} .
$$

Therefore we have

$$
\int_{\mathbf{R}^{4}}\left|\Delta \omega_{k}\right|^{2}+\left|\nabla \omega_{k}\right|^{4} \leq C k^{-1}
$$


Hence, Theorem 2.2 implies that for $k$ sufficiently large, we have

$$
R^{4} \max _{B_{R}}\left(\left|\Delta \omega_{k}\right|^{2}+\left|\nabla \omega_{k}\right|^{4}\right) \leq C k^{-1}, \forall R>0 .
$$

Letting $R$ tend to infinity, we have that $\omega_{k}$ is constant, for $k$ sufficiently large. This contradicts with the choice of $\omega_{k}$. Therefore (2.20) is true.

Related to the lower bound estimate of $\epsilon_{0}$, we have the following conjecture.

Conjecture 2.4. For $N \subset \mathbf{R}^{k}$ of dimension 4 , we have

$$
\begin{aligned}
\epsilon_{0}^{2} & =\inf \left\{\int_{\mathbf{R}^{4}}|\Delta \omega|^{2}: \omega \in C^{\infty} \cap W^{2,2}\left(\mathbf{R}^{4}, N\right) \text { nonconstant biharmonic }\right\} \\
& \geq 4 S(4)^{2} \sqrt{\left|S^{4}\right|},
\end{aligned}
$$

where $S(4)$ is the best Sobolev constant of $W^{1,2}\left(\mathbf{R}^{4}\right) \subset L^{4}\left(\mathbf{R}^{4}\right)$.

Our motivation for (2.24) is follows. Since $\omega \in C^{\infty} \cap W^{2,2}\left(\mathbf{R}^{4}, N\right)$ is biharmonic map, it follows from removability of isolated singularities (see $[\mathrm{W} 1,2]$ ) that $\lim _{|x| \rightarrow \infty} \omega(x)$ exists and $\omega: \mathbf{R}^{4} \rightarrow N$ has a well-defined topological degree. Moreover, by the Sobolev embedding inequality, the geometry-mean inequality, and the integral representation formula of degree, we have

$$
\begin{aligned}
\int_{\mathbf{R}^{4}}|\Delta \omega|^{2} & =\int_{\mathbf{R}^{4}}\left|\nabla^{2} \omega\right|^{2} \geq S(4)^{2}\left(\int_{\mathbf{R}^{4}}|\nabla \omega|^{4}\right)^{\frac{1}{2}} \\
& \geq 4 S(4)^{2} \sqrt{\left|\int_{\mathbf{R}^{4}} \operatorname{det}(\nabla \omega)\right|} \\
& =4 S(4)^{2} \sqrt{|\operatorname{deg}(\omega)|\left|S^{4}\right|}
\end{aligned}
$$

where $\left|S^{4}\right|$ denotes the volume of the unit sphere $S^{4}$. Hence, if we can prove that any nonconstant extrinsic biharmonic map $\omega \in C^{\infty} \cap W^{2,2}\left(\mathbf{R}^{4}, N\right)$ has nonzero degree, then (2.24) holds. However, this is unknown.

We end this section with a well-known result on the density theorem (see, for example, Brezis-Nirenberg [BN]).

Theorem 2.5. If the dimension of $M$ is 4 , then $C^{\infty}(M, N)$ is dense in $W^{2,2}(M, N)$ with respect to $W^{2,2}$-norm.

$\S 3$. Lamm's integral estimates and proof of Theorem A

In this section, we first recall some integral estimates for smooth solutions of (1.3)-(1.4), which are essentially due to Lamm [L2]. Then, by combining Theorem 2.2 and 2.4, we give a proof of Theorem A.

Throughout this section, we asssume $\operatorname{dim} M=4$ and denote by $\operatorname{inj}(M)$ the injectivity radius of $M$. For $0<r<\operatorname{inj}(M)$, denote by $B_{r}(x) \subset M$ the geodesic ball of radius $r$ and center $x$. We start with 
Theorem 3.1. There exists a $\epsilon_{1}>0$, depending only on $M, N$, such that for $0<T<\infty$, if $u \in C^{\infty}(M \times(0, T), N)$ is a solution of (1.3)-(1.4) satisfying

$$
\sup _{0<t<T} \sup _{x \in M} \int_{B_{r_{0}}(x)}|\nabla u|^{4} \leq \epsilon_{1}^{2},
$$

for some $0<r_{0}<\operatorname{inj}(M)$, then we have

$$
\max _{\frac{T}{2} \leq t \leq T}\|u\|_{C^{k}(M)} \leq C\left(k, \epsilon_{1}, r_{0}^{-1}, T,\left\|\nabla^{2} \phi\right\|_{L^{2}(M)}\right), \forall k \geq 1 .
$$

For the convenience of the readers, we outline some key Lemmas needed in the proof of Theorem 3.1. The reader can consult [L2] for more details.

Lemma 3.2. For $T>0$, if $u \in C^{\infty}(M \times(0, T), N)$ solves (1.3)-(1.4), then we have

$$
\int_{0}^{T} \int_{M}\left|u_{t}\right|^{2}+\int_{M}|\Delta u|^{2}(\cdot, T) \leq \int_{M}|\Delta \phi|^{2} .
$$

Moreover, for any $\eta \in C_{0}^{\infty}(M)$,

$$
\begin{aligned}
\int_{0}^{T} \int_{M} \eta^{4}\left|u_{t}\right|^{2} & +\int_{M} \eta^{4}|\Delta u|^{2}(x, T) \leq C \int_{0}^{T} \int_{M}\left(|\Delta \eta|^{2}+|\nabla \eta|^{4}\right)|\Delta u|^{2} \\
& +\int_{M} \eta^{4}|\Delta \phi|^{2}+C \int_{0}^{T} \int_{M}|\nabla \eta|^{2}|\nabla \Delta u|^{2} .
\end{aligned}
$$

Proof. Multiplying (1.3) by $u_{t}$, integrating the resulting equation over $M$, using $u_{t}+\Delta^{2} u \perp T_{u} N$, and then integrating it over $[0, T]$, one can obtain (3.3). For (3.4), multiplying (1.3) by $\eta^{4} u_{t}$, integrating over $M$, using integration by parts and the Hölder inequality, one has

$$
\begin{aligned}
\int_{M} \eta^{4}\left|u_{t}\right|^{2} & +\frac{1}{2} \frac{d}{d t} \int_{M} \eta^{4}|\Delta u|^{2}=\int_{M} \Delta \eta^{4}\left\langle\Delta u, u_{t}\right\rangle+2 \int_{M}\left\langle\nabla \Delta u, u_{t}\right\rangle \cdot \nabla \eta^{4} \\
& =4 \int_{M} \eta^{2}\left(\eta \Delta \eta+3|\nabla \eta|^{2}\right)\left\langle\Delta u, u_{t}\right\rangle+8 \int_{M} \eta^{3}\left\langle\nabla \Delta u, u_{t}\right\rangle \cdot \nabla \eta \\
& \leq \frac{1}{4} \int_{M} \eta^{4}\left|u_{t}\right|^{2}+C \int_{M}\left(|\Delta \eta|^{2}+|\nabla \eta|^{4}\right)|\Delta u|^{2}+|\nabla \eta|^{2}|\nabla \Delta u|^{2}
\end{aligned}
$$

Integrating this over $t \in(0, T)$, we get (3.4).

Now we need to establish $W^{4,2}$-estimate for $u$. More precisely,

Lemma 3.3. There exists an $\epsilon_{1}>0$ depending only on $M, N$, such that for $T>0$, if $u \in C^{\infty}(M \times(0, T), N)$ is a solution of (1.3)-(1.4) satisfying

$$
\sup _{0<t<T} \sup _{x \in M} \int_{B_{r_{0}}(x)}|\nabla u|^{4} \leq \epsilon_{1}^{2}
$$

for some $0<r_{0}<\operatorname{inj}(M)$, then we have

$$
\int_{M}\left|\nabla^{4} u\right|^{2} \leq C\left(\int_{M}\left|u_{t}\right|^{2}+r_{0}^{-4} \int_{M}\left|\nabla^{2} \phi\right|^{2}\right) .
$$


Proof. For any $B_{r_{0}}(x) \subset M$, let $\eta \in C_{0}^{\infty}\left(B_{r_{0}}(x)\right)$ be such that $0 \leq \eta \leq 1$, $\eta=1$ on $B_{\frac{r_{0}}{2}}(x),|\nabla \eta| \leq 4 r_{0}^{-1}$, and $\left|\nabla^{2} \eta\right| \leq C r_{0}^{-2}$. Multiplying (1.3) by $\eta^{4} \Delta^{2} u$, integrating over $M$, and applying Hölder inequality, we have

$$
\begin{aligned}
\int_{M} \eta^{4}\left|\Delta^{2} u\right|^{2} \leq C\left[\int_{M} \eta^{4}\left|u_{t}\right|^{2}\right. & +\int_{M} \eta^{4}\left(|\Delta u|^{4}+|\Delta u|^{2}|\nabla u|^{4}\right. \\
& \left.\left.+|\nabla \Delta u|^{2}|\nabla u|^{2}+|\nabla u|^{8}\right)\right] .
\end{aligned}
$$

Applying the interpolation inequalities (see [L2] Lemma 2.4), we have

$$
\begin{aligned}
\int_{M} \eta^{4}|\Delta u|^{4} & \leq C\left(\int_{B_{r_{0}}(x)}|\nabla u|^{4}\right)^{\frac{1}{2}}\left(\int_{M} \eta^{4}\left|\nabla^{4} u\right|^{2}+\left|\nabla^{2} \eta\right|^{2}\left|\nabla^{2} u\right|^{2}\right) \\
& \leq C \epsilon_{1}\left(\int_{M} \eta^{4}\left|\nabla^{4} u\right|^{2}+\left|\nabla^{2} \eta\right|^{2}\left|\nabla^{2} u\right|^{2}\right) .
\end{aligned}
$$

Similarly, one has

$$
\begin{gathered}
\int_{M} \eta^{4}|\nabla u|^{2}|\nabla u|^{4} \leq C \epsilon_{1}\left(\int_{M} \eta^{4}\left|\nabla^{4} u\right|^{2}+\left|\nabla^{2} \eta\right|^{2}\left|\nabla^{2} u\right|^{2}\right), \\
\int_{M} \eta^{4}|\nabla \Delta u|^{2}|\nabla u|^{2} \leq C \epsilon_{1}\left(\int_{M} \eta^{4}\left|\nabla^{4} u\right|^{2}+\left|\nabla^{2} \eta\right|^{2}\left|\nabla^{2} u\right|^{2}\right), \\
\int_{M} \eta^{4}|\nabla u|^{8} \leq C \epsilon_{1}\left(\int_{M} \eta^{4}\left|\nabla^{4} u\right|^{2}+\left|\nabla^{2} \eta\right|^{2}\left|\nabla^{2} u\right|^{2}\right) .
\end{gathered}
$$

Putting (3.7)-(3.10) into (3.6) and choosing $\epsilon_{1}$ to be sufficiently small, we have

$$
\int_{M} \eta^{4}\left|\nabla^{4} u\right|^{2} \leq C\left(\int_{M} \eta^{4}\left|u_{t}\right|^{2}+\left(|\nabla \eta|^{4}+\left|\nabla^{2} \eta\right|^{2}\right)\left|\nabla^{2} u\right|^{2}\right) .
$$

This implies

$$
\int_{B_{\frac{r_{0}}{2}(x)}}\left|\nabla^{4} u\right|^{2} \leq C\left(\int_{B_{r_{0}}(x)}\left|u_{t}\right|^{2}+r_{0}^{-4} \int_{B_{r_{0}}(x)}\left|\nabla^{2} u\right|^{2}\right) .
$$

Now we can apply the Vitali's covering Lemma to get

$$
\int_{M}|\nabla u|^{4} \leq C\left(\int_{M}\left|u_{t}\right|^{2}+r_{0}^{-4} \int_{M}\left|\nabla^{2} u\right|^{2}\right) .
$$

This, combined with (3.3), implies (3.5).

Now we need to have the uniform control of $\int_{M}\left|u_{t}\right|^{2}$. For this, we recall the following Lemma, whose proof can be found in [L2] Lemma 3.6.

Lemma 3.4. There exists an $\epsilon_{1}>0$ depending only on $M, N$ such that if $u \in$ $C^{\infty}(M \times(0, T), N)$ solves (1.3)-(1.4) and satisfies

$$
\sup _{0<t<T} \sup _{x \in M} \int_{B_{r_{0}}(x)}|\nabla u|^{4} \leq \epsilon_{1}^{2}
$$


for some $0<r_{0}<\operatorname{inj}(M)$. Then there exist $\beta \in\left(0, \frac{1}{2}\right)$ and $0<\delta<\min \left\{T, \beta r_{0}^{4}\right\}$ such that for any $0<s<t<T$, with $|t-s| \leq \delta$, we have

$$
\int_{M}\left|u_{t}\right|^{2}(\cdot, s) \leq C\left(1+\int_{M}\left|u_{t}\right|^{2}(\cdot, t)\right),
$$

where $C=C\left(\int_{M}\left|\nabla^{2} \phi\right|^{2}, M, N\right)>0$.

\section{Proof of Theorem 3.1.}

It follows from Lemma 3.3 and 3.4 that $\nabla^{4} u \in L^{\infty}\left(\left[\frac{T}{4}, T\right], L^{2}(M)\right)$. Hence, by the Sobolev embedding theorem, we conclude that $u_{t}+\Delta^{2} u \in L^{p}\left(M \times\left[\frac{T}{4}, T\right]\right)$ for any $1<p<\infty$. Therefore, by the parabolic $L^{p}$ theory and Schauder estimate, we can achieve the desired estimate (3.2).

To prove Theorem A, we also need the following estimate on the lower bound of the time interval in which smooth solutions of (1.3)-(1.4) exist.

Lemma 3.5. There exist $0<\epsilon_{2}<<\epsilon_{1}$ and $\beta_{0} \in\left(0, \frac{1}{4}\right)$ such that if $\phi \in$ $C^{\infty}(M, N)$ satisfies

$$
\sup _{x \in M} \int_{B_{2 r_{0}}(x)}\left|\nabla^{2} \phi\right|^{2} \leq \epsilon_{2}^{2},
$$

for some $0<r_{0}<\frac{i n j(M)}{2}$. Then there exist $T_{0} \geq \beta_{0} r_{0}^{4}$ and $u \in C^{\infty}\left(M \times\left[0, T_{0}\right], N\right)$ solving (1.3)-(1.4).

Proof. Let $T_{0}>0$ be the maximum such that there exists a smooth solution $u \in C^{\infty}\left(M \times\left[0, T_{0}\right], N\right)$ of (1.3)-(1.4). Let $0 \leq t_{0} \leq T_{0}$ be the maximum such that

$$
\sup _{0<s<t_{0}} \sup _{x \in M} \int_{\frac{B_{\frac{3 r_{0}}{2}}(x)}{}|\nabla u|^{4} \leq 2 \epsilon_{2}^{2}}
$$

Note that (3.15) implies $t_{0}>0$. It follows from (3.4), Lemma 3.3, Lemma 3.4 that for any $x \in M$,

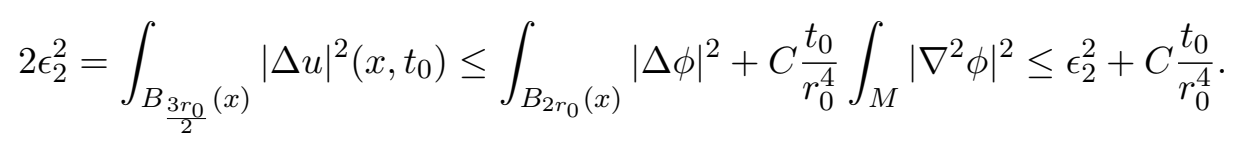

This implies $t_{0} \geq \frac{\epsilon_{2}^{2}}{C r_{0}^{4}}$. This, combined with the Sobolev inequality and (3.16), implies

$$
\sup _{0<t<\frac{\epsilon_{2}^{2}}{C r_{0}^{4}}} \sup _{x \in M} \int_{B_{r_{0}}(x)}|\nabla u|^{4} \leq C \epsilon_{2}^{2} \leq \epsilon_{1}^{2} .
$$

Hence Theorem 3.1 implies the conclusion of Lemma 3.5.

\section{Proof of Theorem A.}


Note that (3) of Theorem A follows from Theorem 2.2. For $\phi \in W^{2,2}(M, N)$, it follows from Theorem 2.5 that there exist $\phi_{n} \in C^{\infty}(M, N)$ such that $\lim _{n \rightarrow \infty} \| \phi_{n}-$ $\phi \|_{W^{2,2}(M)}=0$. Hence there exists a $r_{0} \in\left(0, \frac{\operatorname{inj}(M)}{2}\right)$ such that

$$
\sup _{n} \sup _{x \in M} \int_{B_{2 r_{0}}(x)}\left|\Delta \phi_{n}\right|^{2} \leq \epsilon_{2}^{2}
$$

where $\epsilon_{2}>0$ is the same constant as in Lemma 3.5. Let $u_{n} \in C^{\infty}(M \times$ $\left.\left[0, T_{n}\right], N\right)$ be smooth solutions to (1.3) under the initial condition $u_{n}(x, 0)=$ $\phi_{n}(x)$. Then Lemma 3.5 implies $T_{n} \geq \beta_{0} r_{0}^{4}$. Moreover, Theorem 3.1 implies that we have uniform $C^{k}$-estimates of $u_{n}$ in $M \times\left[0, \beta_{0} r_{0}^{4}\right]$. Hence, after taking possible subsequence, we can assume that $u_{n} \rightarrow u$ weakly in $W^{2,2}(M, N)$, strongly in $W^{1,2}(M, N)$, and in $C^{k}\left(M \times\left[\delta, \beta_{0} r_{0}^{4}\right]\right)$ for any $\delta>0$. It is clear that $u \in C^{\infty}\left(M \times\left(0, \beta_{0} r_{0}^{4}\right), N\right)$ is a solution of $(1.3)$ and satisfies $u(x, 0)=\phi(x)$ in the sense of trace. Now we assume that $T_{0} \geq \beta_{0} r_{0}^{4}$ is the maximum time such that $u \in C^{\infty}\left(M \times\left(0, T_{0}\right), N\right)$ solves (1.3)-(1.4). It follows from Theorem 3.1 that $T_{0}$ can be characterized by

$$
\lim _{r \rightarrow 0} \limsup _{t \uparrow T_{0}} \sup _{x \in M} \int_{B_{r}(x)}|\nabla u|^{4}(\cdot, t) \geq \epsilon_{1}^{2} .
$$

Now we claim that there exists an $\hat{\epsilon}_{0} \geq \epsilon_{0}$ such that

$$
\lim _{r \rightarrow 0} \limsup _{t \uparrow T_{0}} \sup _{x \in M} \int_{B_{r}(x)}|\Delta u|^{2}(\cdot, t) \geq \hat{\epsilon}_{0}^{2} .
$$

The proof of (3.18) is given at the end of the proof. For the moment, assume that (3.18) is true, we want to show that the Hessian energy drops at least $\hat{\epsilon}_{0}^{2}$ at $T_{0}$. In fact, it follows from (3.3) that there is a well-defined trace $u\left(x, T_{0}\right)=$ $\lim _{t \uparrow T} u(x, T)$ weakly in $W^{2,2}(M, N)$. In particular, $u\left(\cdot, T_{0}\right) \in W^{2,2}(M, N)$. Moreover, (3.18) implies that there exists $\left\{x_{i}\right\} \subset M$, with $x_{i} \rightarrow x_{0} \in M, r_{i} \downarrow 0$, and $t_{i} \uparrow T_{0}$ such that

Now we claim

$$
\lim _{i \rightarrow \infty} \int_{B_{r_{i}}\left(x_{i}\right)}|\Delta u|^{2}\left(\cdot, t_{i}\right) \geq \hat{\epsilon}_{0}^{2}
$$

$$
\int_{M}|\Delta u|^{2}\left(\cdot, T_{0}\right) \leq \int_{M}|\Delta \phi|^{2}-\hat{\epsilon}_{0}^{2}
$$

In fact, by the lower semicontinuity and (3.3), we have, for any $r>0$,

$$
\begin{aligned}
\int_{M \backslash B_{r}\left(x_{0}\right)}|\Delta u|^{2}\left(\cdot, T_{0}\right) & \leq \liminf _{i \rightarrow \infty} \int_{M \backslash B_{r}\left(x_{0}\right)}|\Delta u|^{2}\left(\cdot, t_{i}\right) \\
& =\liminf _{i \rightarrow \infty}\left(\int_{M}|\Delta u|^{2}\left(\cdot, t_{i}\right)-\int_{B_{r}\left(x_{0}\right)}|\Delta u|^{2}\left(\cdot, t_{i}\right)\right) \\
& \leq \liminf _{i \rightarrow \infty} \int_{M}|\Delta u|^{2}\left(\cdot, t_{i}\right)-\lim _{i \rightarrow \infty} \int_{B_{r_{i}}\left(x_{i}\right)}|\Delta u|^{2}\left(\cdot, t_{i}\right)
\end{aligned}
$$




$$
\leq \int_{M}|\Delta \phi|^{2}-\hat{\epsilon}_{0}^{2}
$$

Taking $r$ into zero, (3.21) yields (3.20).

Now we use $u\left(\cdot, T_{0}\right)$ as the initial data to extend the above solution beyond $T_{0}$ to obtain a weak solution $u: M \times\left(0, T_{1}\right) \rightarrow N$ of (1.3)-(1.4) for some $T_{1}>T_{0}$. Since $H(u(t))$ drops at least $\hat{\epsilon}_{0}^{2}$ at each singular time, we have that after at most $\left[\frac{\int_{M}|\Delta \phi|^{2}}{\hat{\epsilon}_{0}^{2}}\right]$-times, the solution can be extended smoothly to be a global weak solution that satisfies (1.5) and (1.6) with $\epsilon_{0}$ replaced by $\hat{\epsilon}_{0}$.

To prove (4), we proceed as follows (see also [L2] Theorem 1.1). For $1 \leq i \leq$ $L-1, t \in\left(t_{i}, t_{i+1}\right)$, and $0<r<\operatorname{inj}(M)$, define the concentration function

$$
Q(t, r)=\sup \left\{\int_{B_{r}(x)}\left(|\Delta u|^{2}+|\nabla u|^{4}\right)(z, \tau) d z \mid B_{r}(x) \subset M, t_{i} \leq \tau \leq t\right\}
$$

Then we have (i) $Q(t, r)$ is monotonically nondecreasing and continuous w.r.t. $r>0$, (ii) $Q(t, 0)=0$, and (iii) $Q(t, r)$ is monotonically nondecreasing w.r.t. $t<t_{i+1}$, and

$$
\lim _{t \uparrow t_{i+1}} Q(t, r) \geq \epsilon_{1}^{2},
$$

where $\epsilon_{1}$ is given by (3.17). Therefore, we can conclude that there exist $t_{n} \uparrow t_{i+1}$, $r_{n} \downarrow 0$, and $x_{n} \rightarrow x_{0} \in M$ such that

$$
Q\left(t_{n}, r_{n}\right)=\frac{\epsilon_{1}^{2}}{C_{0}}=\int_{B_{r_{n}}\left(x_{n}\right)}\left(|\Delta u|^{2}+|\nabla u|^{4}\right)\left(\cdot, t_{n}\right)
$$

where $C_{0}>0$ is to be determined. (3.22) implies, for some $\delta_{0}>0$,

$$
\int_{B_{r_{n}}(x)}\left(|\Delta u|^{2}+|\nabla u|^{4}\right)\left(\cdot, t_{n}\right) \leq \frac{\epsilon_{1}^{2}}{C_{0}}, \forall B_{r_{n}}(x) \subset B_{\delta_{0}}\left(x_{0}\right) .
$$

This and Lemma 3.5 imply

$$
\int_{B_{r_{n}}(x)}\left(\left|\nabla^{2} u\right|^{2}+|\nabla u|^{4}\right)(\cdot, t) \leq \frac{C \epsilon_{1}^{2}}{C_{0}} \leq \epsilon_{1}^{2}, \quad \forall x \in B_{\frac{\delta_{0}}{2}}\left(x_{0}\right), \quad t_{n} \leq t \leq t_{n}+\beta_{0} r_{n}^{4}
$$

provided that $C_{0}$ is sufficiently large. Define $u_{n}(x, t)=u\left(x_{n}+r_{n} x, t_{n}+r_{n}^{4} t\right) \in$ $C^{\infty}\left(B_{r_{n}^{-1} \delta_{0}} \times\left[r_{n}^{-4}\left(t_{i}-t_{n}\right), \beta_{0}\right], N\right)$. Then $u_{n}$ are smooth solutions of (1.3). Applying Theorem 3.1 to $u_{n}$, we conclude that, after taking possible subsequences, $u_{n} \rightarrow \omega$ in $C_{\mathrm{loc}}^{k}\left(\mathbf{R}^{4} \times\left(-\infty, \beta_{0}\right)\right)$ for any $k \geq 4$. Moreover, $\left\|\frac{\partial u_{n}}{\partial t}\right\|_{L^{2}} \rightarrow 0$ implies $\omega \in C^{\infty} \cap W^{2,2}\left(\mathbf{R}^{4}, N\right)$ is a nontrivial biharmonic map. This proves (4).

Now we return to the proof of (3.18). For any $\epsilon>0$, let $R>0$ such that

$$
\int_{B_{R}}|\Delta \omega|^{2} \geq \int_{\mathbf{R}^{4}}|\Delta \omega|^{2}-\epsilon
$$


Then we have, for any $r>0$,

$$
\begin{aligned}
\epsilon_{0}^{2}-\epsilon & \leq \int_{\mathbf{R}^{4}}|\Delta \omega|^{2}-\epsilon \leq \int_{B_{R}}|\Delta \omega|^{2} \\
& =\lim _{n \rightarrow \infty} \int_{B_{R}}\left|\Delta u_{n}\right|^{2}(\cdot, 0) \\
& =\lim _{n \rightarrow \infty} \int_{B_{R_{n}}\left(x_{n}\right)}|\Delta u|^{2}\left(\cdot, t_{n}\right) \\
& \leq \limsup _{t \uparrow T_{0}} \sup _{x \in M} \int_{B_{r}(x)}|\Delta u|^{2}(\cdot, t) .
\end{aligned}
$$

Since $\epsilon$ is arbitrary, this clearly implies (3.18). Hence the proof of Theorem A is complete.

\section{$\S 4$ Proof of Theorem B}

This section is devoted to the proof of Theorem B.

\section{Proof of Theorem B.}

The aim is to prove that the global weak solution obtained by Theorem A is smooth, provided that the initial data is properly chosen. Our idea is similar to that of Struwe [Sm3] on the heat flow of harmonic maps from surfaces.

Let $\epsilon_{0}>0$ be given by (1.7). For any $\epsilon<\frac{\epsilon_{0}}{2}$ and $\alpha \in[M, N]$, let $u_{0} \epsilon$ $\alpha \cap C^{\infty}(M, N)$ be such that

$$
\int_{M}\left|\Delta u_{0}\right|^{2}-\epsilon^{2} \leq C_{\alpha} \equiv \inf _{v \in \alpha \cap C^{2}(M, N)} \int_{M}|\Delta v|^{2}
$$

and $u: M \times \mathbf{R}_{+} \rightarrow N$ be the global weak solution of $(1.3)$, with $u(x, 0)=u_{0}(x)$ for $x \in M$, given by Theorem A. We want to show that $u \in C^{\infty}(M \times[0,+\infty), N)$. For otherwise, let $0<T_{0}<+\infty$ be the first singular time of $u$. By (4) of Theorem A, we know that there exist $x_{n} \rightarrow x_{0} \in M, t_{n} \uparrow T_{0}$, and $\lambda_{n} \downarrow 0$, and a bubble $\omega \in C^{\infty} \cap W^{2,2}\left(\mathbf{R}^{4}, N\right)$ such that

$$
u_{n}(\cdot) \equiv u\left(x_{n}+\lambda_{n} \cdot t_{n}\right) \rightarrow \omega, \text { in } C_{\mathrm{loc}}^{4}\left(\mathbf{R}^{4}, N\right) .
$$

For any $C_{0}>0$, let $R=R\left(C_{0}\right)>0$ be so large that

$$
\int_{R^{4} \backslash B_{R}}|\Delta \omega|^{4}+|\nabla \omega|^{4} \leq \frac{\epsilon_{0}^{2}}{C_{0}}
$$

Define $\omega_{R}: B_{R} \rightarrow N$ by $\omega_{R}(x)=\omega\left(\frac{R^{2}}{|x|^{2}} x\right), x \in B_{R}$. It follows by removability of isolated singularities (see [W1,2]) that $\hat{\omega}=\omega \circ \Phi \in C^{\infty}\left(S^{4}, N\right)$, where $\Phi: S^{4} \rightarrow$ $\mathbf{R}^{4}$ is the stereographic projection, and hence $\omega_{R} \in C^{\infty}\left(B_{R}, N\right)$.

Let $\eta \in C_{0}^{\infty}\left(B_{R}\right)$ be such that $0 \leq \eta \leq 1, \eta \equiv 0$ in $B_{\frac{R}{4}}, \eta \equiv 1$ on $B_{R} \backslash B_{\frac{R}{2}}$, $|\nabla \eta| \leq 8 R^{-1}$, and $\left|\nabla^{2} \eta\right| \leq C R^{-2}$. Define $v_{n}(x)=(1-\eta(x)) \omega_{R}(x)+\eta u_{n}(x), x \in$ 
$B_{R}$. Then we have

$$
\begin{aligned}
\max _{B_{R}} \operatorname{dist}\left(v_{n}(x), N\right) & \leq \max _{B_{R} \backslash B_{\frac{R}{4}}}\left|(1-\eta(x)) \omega_{R}(x)+\eta u_{n}(x)-\omega_{R}(x)\right| \\
& \leq \max _{B_{R} \backslash B_{\frac{R}{4}}}\left|\omega_{R}(x)-u_{n}(x)\right| \\
& \leq \max _{B_{R} \backslash B_{\frac{R}{4}}}\left(\left|\omega_{R}(x)-\omega(x)\right|+\left|u_{n}(x)-\omega(x)\right|\right) \\
& \leq \operatorname{osc}_{B_{4 R} \backslash B_{\frac{R}{4}}} \omega+o(1)=o\left(1, R^{-1}\right)
\end{aligned}
$$

where $\lim _{n, R \rightarrow \infty} o\left(1, R^{-1}\right)=0$. Therefore for any $\delta>0, v_{n}\left(B_{R}\right) \subset N_{\delta}$ for sufficiently large $R$ and $n$. For $\delta>0$ sufficiently small, let $\Pi: N_{\delta} \rightarrow N$ be the smooth nearest point projection map, and define $w_{n}: B_{R} \rightarrow N$ by $w_{n}(x)=$ $\Pi\left(v_{n}(x)\right), x \in B_{R}$, and $\bar{w}_{n}: M \rightarrow N$ by

$$
\begin{aligned}
\bar{w}_{n}(x) & =w_{n}\left(\frac{x-x_{n}}{\lambda_{n}}\right), x \in B_{\lambda_{n} R}\left(x_{n}\right) \\
& =u\left(x, t_{n}\right), x \in M \backslash B_{\lambda_{n} R}\left(x_{n}\right) .
\end{aligned}
$$

It is clear that $\bar{w}_{n} \in C^{\infty}(M, N)$. Moreover, since $\Pi_{4}(N)=\{0\}$, it follows that $\bar{w}_{n} \in \alpha$.Therefore we have

$$
\begin{aligned}
& C_{\alpha} \leq \int_{M}\left|\Delta \bar{w}_{n}\right|^{2} \\
& =\int_{M \backslash B_{\lambda_{n} R}\left(x_{n}\right)}|\Delta u|^{2}\left(\cdot, t_{n}\right)+\int_{B_{\lambda_{n} R}\left(x_{n}\right)}\left|\Delta \bar{w}_{n}\right|^{2} \\
& =\int_{M}|\Delta u|^{2}\left(\cdot, t_{n}\right)-\int_{B_{\lambda_{n} R}\left(x_{n}\right)}|\Delta u|^{2}\left(\cdot, t_{n}\right)+\int_{B_{\lambda_{n} R}\left(x_{n}\right)}\left|\Delta \bar{w}_{n}\right|^{2} .
\end{aligned}
$$

Note that, by (4.2) and change of variables, we have, for sufficiently large $n, R$,

$$
\int_{B_{\lambda_{n} R}\left(x_{n}\right)}|\Delta u|^{2}\left(\cdot, t_{n}\right)=\int_{B_{R}}\left|\Delta_{g_{n}} u_{n}\right|^{2} d v_{g_{n}} \geq \frac{3}{4} \int_{B_{R}}|\Delta \omega|^{2} \geq \frac{\epsilon_{0}^{2}}{2},
$$

where $g_{n}(x)=g\left(x_{n}+\lambda_{n} x\right), x \in B_{R}$ and $\Delta_{g_{n}}$ is the Laplace operator w.r.t. $g_{n}$. On the other hand, direct calculations imply

$$
\begin{aligned}
& \int_{B_{\lambda_{n} R}\left(x_{n}\right)}\left|\Delta \bar{w}_{n}\right|^{2}=\int_{B_{R}}\left|\Delta_{g_{n}} w_{n}\right|^{2} d v_{g_{n}} \\
& \leq C \int_{B_{R}}\left|\Delta_{g_{n}}\left((1-\eta) \omega_{R}+\eta u_{n}\right)\right|^{2} d v_{g_{n}} \\
& =C \int_{B_{\frac{R}{4}}}\left|\Delta_{g_{n}} \omega_{R}\right|^{2} d v_{g_{n}} \\
& +C \int_{B_{R} \backslash B_{\frac{R}{4}}}\left|\Delta_{g_{n}}\left((1-\eta) \omega_{R}+\eta u_{n}\right)\right|^{2} d v_{g_{n}} \\
& \leq C \int_{R^{4} \backslash B_{R}}\left(|\Delta \omega|^{2}+|\nabla \omega|^{4}\right) \\
& +C \int_{B_{R} \backslash B_{\frac{R}{4}}}\left(\left|\Delta \omega_{R}\right|^{2}+\left|\Delta u_{n}\right|^{2}+R^{-4}\left|u_{n}-\omega_{R}\right|^{2}+R^{-2}\left|\nabla\left(u_{n}-\omega_{R}\right)\right|^{2}\right) \\
& \leq \frac{C \epsilon_{0}^{2}}{C_{0}}+o\left(1, R^{-1}\right) \leq \frac{\epsilon_{0}^{2}}{4}+o\left(1, R^{-1}\right) \leq \frac{\epsilon_{0}^{2}}{2}
\end{aligned}
$$


provided that $C_{0}, n, R$ are sufficiently large. Therefore, by (1.5) and (4.4)-(4.6), we have

$$
C_{\alpha} \leq \int_{M}|\Delta u|^{2}\left(\cdot, t_{n}\right)-\frac{\epsilon_{0}^{2}}{2} \leq \int_{M}\left|\Delta u_{0}\right|^{2}-\frac{\epsilon_{0}^{2}}{2} .
$$

This contradicts with (4.1). Hence $u \in C^{\infty}(M \times[0,+\infty), N)$.

Next we want to prove that there exists $t_{n} \rightarrow \infty$ and a smooth biharmonic map $u_{\infty} \in C^{\infty}(M, N)$ such that $u\left(\cdot, t_{n}\right) \rightarrow u_{\infty}$ in $C^{2}(M, N)$.

To prove this, we first observe that (1.5) implies there exist $t_{n} \uparrow+\infty$ such that $\left\|u_{t}\left(\cdot, t_{n}\right)\right\|_{L^{2}(M)} \rightarrow 0$ and there exists $u_{\infty} \in W^{2,2}(M, N)$ such that $u\left(\cdot, t_{n}\right) \rightarrow u_{\infty}$ weakly in $W^{2,2}(M, N)$. Moreover, $u\left(\cdot, t_{n}\right) \rightarrow u_{\infty}$ in $C^{k}(M \backslash \Sigma, N)$ for any $k \geq 1$, where $\Sigma \subset M$ is given by

$$
\Sigma=\cap_{r>0}\left\{\left.x \in M\left|\liminf _{n \rightarrow \infty} \int_{B_{r}(x)}\right| \nabla u\right|^{4}\left(\cdot, t_{n}\right) \geq \epsilon_{1}^{2}\right\}
$$

where $\epsilon_{1}$ is the same constant as in Theorem 3.1. It is easy to see that a simple covering argument implies $\Sigma \subset M$ is a finite set. Theorem 3.1 and Lemma 3.5 imply the smooth convergence of $u\left(\cdot, t_{n}\right)$ to $u_{\infty}$ away from $\Sigma$. This implies that $u_{\infty} \in W^{2,2}(M, N) \cap C^{\infty}(M \backslash \Sigma, N)$ is a biharmonic map. Hence, by the removability of isolated singularities ([W1,2]), we conclude that $u_{\infty} \in C^{\infty}(M, N)$ is a biharmonic map. We may assume that for any given $x_{0} \in \Sigma$, there is a $r_{0}>0$ such that $u\left(\cdot, t_{n}\right) \rightarrow u_{\infty}$ in $C_{\mathrm{loc}}^{k}\left(B_{r_{0}}\left(x_{0}\right) \backslash\left\{x_{0}\right\}, N\right)$.

Now we do a surgery of $u\left(\cdot, t_{n}\right)$ near $x_{0}$ as follows. Let $\eta \in C_{0}^{\infty}\left(B_{r_{0}}\left(x_{0}\right)\right)$ be such that $0 \leq \eta \leq 1, \eta \equiv 0$ in $B_{\frac{r_{0}}{4}}\left(x_{0}\right), \eta \equiv 1$ in $B_{r_{0}}\left(x_{0}\right) \backslash B_{\frac{r_{0}}{2}}\left(x_{0}\right),|\nabla \eta| \leq 8 r_{0}^{-1}$, and $\left|\nabla^{2} \eta\right| \leq C r_{0}^{-2}$. Define $v_{n}: B_{r_{0}}\left(x_{0}\right) \rightarrow N$ by

$$
v_{n}(x)=(1-\eta(x)) u_{\infty}(x)+\eta(x) u\left(x, t_{n}\right), x \in B_{r_{0}}\left(x_{0}\right) .
$$

Then for any $\delta_{0}>0$, there exists $n_{0} \geq 1$ such that for $n \geq n_{0}$, we have

$$
\begin{aligned}
\max _{x \in B_{r_{0}}\left(x_{0}\right)} \operatorname{dist}\left(v_{n}(x), N\right) & \leq \max _{x \in B_{r_{0}}\left(x_{0}\right)}\left|v_{n}(x)-u_{\infty}(x)\right| \\
& \leq \max _{B_{r_{0}}\left(x_{0}\right) \backslash B \frac{r_{0}}{4}\left(x_{0}\right)}\left|u_{\infty}(x)-u\left(x, t_{n}\right)\right| \leq \delta_{0} .
\end{aligned}
$$

Therefore, we can project $v_{n}$ to $N$ to get $w_{n}(x)=\Pi\left(v_{n}(x)\right)$ for $x \in B_{r_{0}}\left(x_{0}\right)$. This gives $\bar{u}_{n} \in C^{\infty}(M, N)$ by

$$
\begin{aligned}
\bar{u}_{n}(x) & =w_{n}(x), \forall x \in B_{r_{0}}\left(x_{0}\right) \\
& =u\left(x, t_{n}\right), \forall x \in M \backslash B_{r_{0}}\left(x_{0}\right) .
\end{aligned}
$$

Since $\Pi_{4}(N)=\{0\}$, we also have $\bar{u}_{n} \in \alpha$ and hence

$$
\begin{aligned}
C_{\alpha} & \leq \int_{M}\left|\Delta \bar{u}_{n}\right|^{2}=\int_{M}|\Delta u|^{2}\left(\cdot, t_{n}\right)-\int_{B_{r_{0}}\left(x_{0}\right)}|\Delta u|^{2}\left(\cdot, t_{n}\right)+\int_{B_{r_{0}}\left(x_{0}\right)}\left|\Delta w_{n}\right|^{2} \\
& \leq \int_{M}|\Delta u|^{2}\left(\cdot, t_{n}\right)-\epsilon_{0}^{2}+\int_{B_{r_{0}}\left(x_{0}\right)}\left|\Delta w_{n}\right|^{2}
\end{aligned}
$$


where we have used the fact that $x_{0} \in \Sigma$ and

$$
\int_{B_{r_{0}}\left(x_{0}\right)}|\Delta u|^{2}\left(\cdot, t_{n}\right) \geq \epsilon_{0}^{2} .
$$

The proof of (4.7) is same as that of (3.18). On the other hand, we have

$$
\begin{aligned}
\int_{B_{r_{0}}\left(x_{0}\right)}\left|\Delta w_{n}\right|^{2} & \leq C \int_{B_{r_{0}}}\left|\Delta v_{n}\right|^{2}+\left|\nabla v_{n}\right|^{4} \\
& \leq C\left(r_{0}\right)\left\{\int_{B_{r_{0}}\left(x_{0}\right) \backslash B_{\frac{r_{0}}{4}}\left(x_{0}\right)}\left(\left|\Delta u_{\infty}\right|^{2}+\left|\nabla u_{\infty}\right|^{4}\right)\right. \\
& +\int_{B_{r_{0}}\left(x_{0}\right) \backslash B \frac{r_{0}}{4}\left(x_{0}\right)}\left(\left|\Delta\left(u_{\infty}-u\left(\cdot, t_{n}\right)\right)\right|^{2}\right. \\
& +\left|u_{\infty}-u\left(\cdot, t_{n}\right)\right|^{2}+\left|\nabla\left(u_{\infty}-u\left(\cdot, t_{n}\right)\right)\right|^{2} \\
& \left.+\mid \nabla\left(u_{\infty}-\left.u\left(\cdot, t_{n}\right)\right|^{4}\right)\right\} \\
& \leq \frac{\epsilon_{0}^{2}}{2}
\end{aligned}
$$

for sufficiently large $t_{n}$. We conclude that $C_{\alpha} \leq C_{\alpha}-\epsilon_{0}^{2}+\frac{\epsilon_{0}^{2}}{2}$. This is impossible. Therefore $u\left(\cdot, t_{n}\right) \rightarrow u_{\infty}$ in $C^{k}(M, N)$. In particular, for any small $\epsilon>0$, there is a biharmonic map $u_{\epsilon} \in C^{\infty}(M, N) \cap \alpha$ such that

$$
\int_{M}\left|\Delta u_{\epsilon}\right|^{2} \leq C_{\alpha}+\epsilon
$$

It can be checked that the same argument as above also yields that there exists $\epsilon_{i} \downarrow 0 u_{\epsilon_{i}} \rightarrow v$ in $C^{k}(M, N)$ for any $k \geq 1$. Hence $v \in C^{\infty}(M, N)$ is a biharmonic map such that $v \in \alpha$ and $\int_{M}|\Delta v|^{2}=C_{\alpha}$. Therefore, there exists a minimizing biharmonic map in $\alpha$. This completes the proof of Theorem B.

\section{REFERENCES}

[BN] H. Brezis, L. Nirenberg, Degree theory and BMO. I. Compact manifolds without boundaries. Selecta Math. (N.S.) 1 (1995) no. 2, 197-263.

[CS] Y. Chen, M. Struwe, Existence and partial regularity results for the heat flow for harmonic maps. Math. Z. 201 (1989), no. 1, 83-103.

[CWY] S. Y. Chang, L. Wang, P. Yang, A regularity theory of biharmonic maps. Comm. Pure Appl. Math. 52 (1999) 1113-1137.

[EL] J. Eells, L. Lemaire, Selected topics in harmonic maps. CBMS Regional Conference Series in Mathematics, 50. American Mathematical Society, Providence, RI, 1983.

[G] A. Gastel, The extrinsic polyharmonic map heat flow in the critical dimension. To appear, Adv. Geom.

[GT] D. Gilbarg, N. Trudinger, Elliptic partial differential equations of second order. Springer, 1983. 
[KS1] E. Kuwert, R. Schätzle, The Willmore flow with small initial energy. J. Differential Geom. 57 (2001) 409-441.

[KS2] E. Kuwert, R. Schätzle, Gradient flow for the Willmore functional. Comm. Anal. Geom. 10(2002), 307-339.

[L1] T. Lamm, Biharmonischer Wärmefluss. Diplomarbeit, Universität Freiburg (2001)

[L2] T. Lamm, Heat flow for extrinsic biharmonic maps with small initial energy. Ann. Global Anal. Geom. 26 (2004), no. 4, 369-384.

[L3] T. Lamm, Biharmonic map heat flow into manifolds of nonpositive curvature. Calc. Var. Partial Differential Equations 22 (2005), no. 4, 421-445.

[Sl] L. Simon, Existence of surfaces minimizing the Willmore functional. Comm. Anal. Geom. 1 (1993), no. 2, 281-326.

[Sm1] M. Struwe, On the evolution of harmonic mappings of Riemannian surfaces. Comm. Math. Helv. 60 (1985) 558-581.

[Sm2] M. Struwe, Heat-flow methods for harmonic maps of surfaces and applications to free boundary problems. Lecture Notes in Math., 1324, 293-319, Springer, Berlin, 1988.

[Sm3] M. Struwe, Geometric Evolution Equations. Nonlinear partial differential equations in differential geometry (Park City, UT, 1992), 257-339. IAS/Park City Math. Ser. 2, Amer. Math. Soc., Providence, RI (1996).

[Sp] P. Strzelecki, On biharmonic maps and their generalizations. Calc. Var. 18 (2003), 401-432.

[Sr] R. Schoen, Analytic aspects of the harmonic map problem. Seminar on nonlinear partial differential equations (Berkeley, Calif., 1983), 321-358, Math. Sci. Res. Inst. Publ., 2, Springer, New York, 1984.

[SU] J. Sacks, K. Uhlenbeck, The existence of minimal immersions of 2-spheres. Ann. of Math. (2) 113 (1981), no. 1, 1-24.

[W1] C. Y. Wang, Biharmonic maps from $\mathbf{R}^{4}$ into a Riemannian manifold. Math. Z. 247 (2004), no. 1, 65-87.

[W2] C. Y. Wang, Stationary biharmonic maps from $\mathbf{R}^{m}$ into a Riemannian manifold. Comm. Pure Appl. Math. 57 (2004), no. 4, 419-444 .

[W3] C. Y. Wang, Remarks on biharmonic maps into spheres. Calc. Var. Partial Differential Equations 21 (2004), no. 3, 221-242. 
[W4] C. Y. Wang, Heat flow of biharmonic maps for small data in dimensions at most 8. In preparation.

Changyou Wang

Department of Mathematics

University of Kentucky, Lexington, KY 40506

E-mail: cywang@ms.uky.edu 